\title{
POISONING-INDUCED EXCHANGE OF STEADY STATES IN A CATALYTIC CHEMICAL REACTOR
}

\author{
D. D. DO and R. H. WEILAND
}

(Received 16 October 1979)

(Revised 24 April 1980)

\begin{abstract}
Slow catalyst poisoning can result in the sudden failure of a chemical reactor operating isothermally with substrate-inhibited kinetics. At failure, a satisfactory steady state is exchanged for one of low conversion. The method of matched asymptotic expansions is used to give a detailed description of the exchange process in the phase plane. The structure of the jump is ascertained by separate asymptotic expansions across two adjoining transition regions in which the independent variables contain unknown shifts.
\end{abstract}

\section{Introduction}

The use of catalysts to promote the rate at which one chemical species is converted to another, more valuable one, is of central importance to the chemical and pharmaceutical industries. In the context of catalysts, we include here not only those of conventional inorganic type but also enzymes, particularly in an immobilized form. A common method of studying the activity of heterogeneous catalysts involves the use of a spinning highly-porous basket which is filled with catalyst and immersed in a well-stirred vessel containing the reactant. This is the so-called constant-flow stirred-tank reactor or CSTR. Thus, the reactant is uniformly distributed throughout the reactor, and within the catalyst particles themselves for highly porous materials. The kinetics can then be studied divorced from diffusional resistances; the equations governing the behaviour of the reactor are ordinary differential equations in time, and we deal with a lumped parameter system.

It is well known that certain classes of chemically reacting systems can exhibit a multiplicity of steady states. Examples include non-isothermal systems obeying Arrhenius chemistry in which the activation energy is sufficiently large [3] and isothermal systems in which the kinetics is of Langmuir-Hinshelwood (substrate 
inhibited) type and the kinetic parameters bear particular relationships [2]. Existence, uniqueness and stability of steady states have been extensively discussed by Aris [3] in the context of heterogeneous catalysis and DeVera and Varma [7] for enzyme catalyzed reactions.

Much of the work reported on the dynamics of the exchange of steady states has been done within the framework of combustion theory, dealing in particular with the so-called ignition phenomenon. Liñan and Williams have calculated the ignition time for a semi-infinite slab of combustible material experiencing a constant energy flux [15] and exposed to a step change in surface temperature [16]. Both lumped and distributed parameter systems have been successfully treated, usually through asymptotic analysis in the limit of large activation energy. In particular, Kapila [11] and Kapila and Matkowsky [13] have treated both the ignition and extinction processes for distributed systems while the spatially homogeneous or lumped parameter equivalent has been examined by Kassoy [14]. All of this work, including that of Buckmaster et al. [5], was done using large activation energy asymptotics and a history of the ignition or extinction event obtained.

Our interest in the current work is in a description of the dynamics of the exchange of steady states in catalytic systems which takes place as a result of the slow passage of one of the system parameters (catalyst activity) through criticality. We deal with a lumped system, governed by a set of ordinary differential equations. Such problems have recently been addressed by Haberman [9] who showed that the transition region is governed by a Riccati equation whose solution could only be matched with one of the steady states. This has been extended to distributed systems in the context of ignition by Kapila [12] who used large activation energy asymptotics. We find a similar state of affairs here.

A common rate expression for heterogeneously catalysed reactions is

$$
R=-\frac{d C_{A}}{d t}=\frac{k C_{A}}{C_{A}^{2}+K_{S} C_{A}+K},
$$

which is the well-known Langmuir-Hinshelwood [17] or substrate inhibited [2] form. Here $C_{A}$ is the concentration of reactant or substrate and $k, K_{S}, K$ are kinetic parameters. Examples of this type of kinetics include carbon monoxide oxidation over platinum as in the automobile catalytic converter, and the reduction of nitrate by Nitrobacter winogradski in the context of biological systems.

Despite their indispensible nature, all catalysts suffer from a common complaint - they slowly become deactivated with continued use over long periods of time. The mechanism of deactivation may involve poisoning by some component present in the feed material in small amounts (impurity poisoning), poisoning by the chemical reactant itself (parallel deactivation) or by some product of the reaction (series poisoning). The latter mechanisms are also referred to as self-poisoning and are common in enzyme systems. As we have pointed out previously [8], a study of 
chemical reaction mechanisms indicates that the catalyst must lose activity according to a rate expression which closely resembles that of the main reaction. Thus, if $a$ represents the activity of a catalyst a typical deactivation rate expression for parallel poisoning is

$$
R_{d}=-\frac{d a}{d t}=\frac{k_{d} C_{A} a}{C_{A}^{2}+K_{S} C_{A}+K}
$$

in which $k_{d}$ is the deactivation rate constant. Activity is a measure of the chemical reaction rate at any time, relative to the rate when the catalyst is fresh. Thus $a \in[0,1]$. When the catalyst is deactivating, the rate constant $k$ in (1.1) must be replaced by $k a$. Thus

$$
R=-\frac{d C_{A}}{d t}=\frac{k C_{A} a}{C_{A}^{2}+K_{S} C_{A}+K}
$$

is the appropriate rate expression for the main reaction.

It is well established that a reactor, in which a reaction is proceeding according to the rate expression (1.1), can operate at any one of a multiplicity of steady states, even under isothermal conditions $[4,10]$. When this is combined with a slow loss in catalyst activity we are faced with the possibility of a reactor suddenly jumping from one pseudo-steady state to another. Suddenly, and without warning, a reactor which has been operating satisfactorily fails and a rapid loss in performance (as measured by the fractional conversion of reactants to products) is experienced.

The equivalent slowly varying parameter in the theory of ignition and extinction may be, for example, the surface temperature or surface energy flux. It must be emphasized, however, that all previous work on the ignition problem has been based on asymptotics in the limit of large activation energy, the phenomenon itself resulting from the interaction of the slowly varying parameter with Arrhenius type nonlinear kinetics. In the present case of an isothermal system, it is the interaction of slowly varying catalyst activity with nonlinear Langmuir-Hinshelwood kinetics, which results in the exchange of steady states. We have no additional large or small parameter equivalent to activation energy about which an asymptotic analysis can be made; the sole parameter at our disposal is a measure of the slowness of deactivation. In a future communication, we shall report on an investigation of the ignition problem when the assumption of large activation energy is relaxed.

The purpose of this paper is to develop solutions to the reactor failure problem which allow prediction of the conditions at failure. We will use the method of matched asymptotic expansions and certain results of bifurcation theory to give a complete description of reactor behaviour throughout its entire time of operation. The events taking place during the jump will be analysed in some detail. 


\section{Formulation and approach}

The transient non-dimensional mass balance equation for the concentration of reactant in a CSTR is

$$
\frac{d A}{d t}=H(1-A)-\frac{A a}{\beta+\gamma A+A^{2}}
$$

and the rate-of-change of catalyst activity is

$$
\frac{d a}{d t}=-\varepsilon \frac{A a}{\beta+\gamma A+A^{2}} .
$$

Here the reactant concentration has been scaled on its value $C_{A_{o}}$ in the feed stream to the reactor, $H$ is the non-dimensional flow rate into the vessel and $\varepsilon$ is the nondimensional deactivation rate constant $\left(k_{d} C_{A_{o}} / k\right)$. We shall assume $\varepsilon \ll 1$; if the rate of deactivation was fast, the catalyst would be of little use. Initially the reactor contains only inert material and the catalyst has unit activity:

$$
\begin{aligned}
& A(0)=0, \\
& a(0)=1 .
\end{aligned}
$$

We shall investigate the system in the phase plane where the appropriate equations are

$$
\begin{gathered}
\varepsilon \frac{d A}{d a}=h(A, a), \\
h(A, a)=1-H(1-A)\left(\beta+\gamma A+A^{2}\right) / A a,
\end{gathered}
$$

with the condition

$$
a=1, A=0 .
$$

When there are multiple steady states the direct application of matched asymptotic expansions in the start-up and deactivating periods breaks down because the outer, or long time, solution possesses two separate branches. At some stage, which we call the branching time, the outer solution jumps discontinuously from one quasi-steady state to the other.

The case of two stable quasi-steady states is shown in Figure 1 where it can be seen that the concentration of reactant, $A$, rises smoothly from a pseudo steady level $A^{+}$, achieved shortly after start-up, to a value $A_{*}$. But because the catalyst slowly continues to be deactivated, the slope of the line marked $H(1-A) / a$ becomes increasingly negative and operation at $A_{*}$ ceases in a very short time. This corresponds to a jump in the solution from the lower branch in which $A^{+}<A<A_{*}$ and $a_{*}<a<1$ to an upper branch where $A>A^{*}$ and $0<a<a_{*}$. The catalyst 


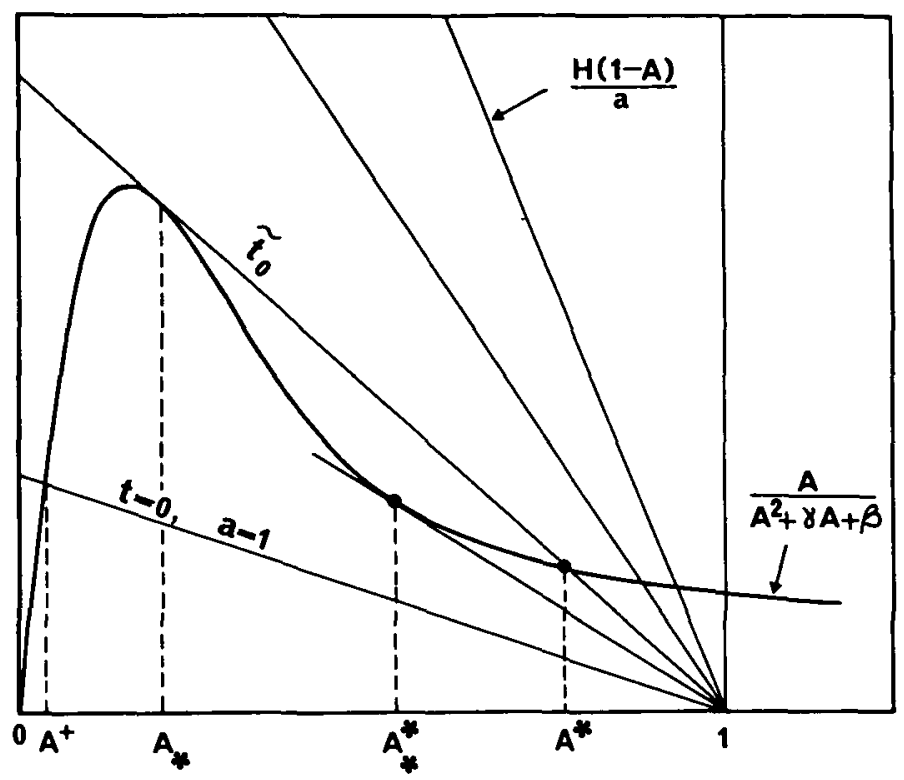

Fig. 1. Schematic of quasi-steady states.

activity does not experience a sudden change because the rate of deactivation is always slow. Thus, when the reactant concentration reaches $A^{*}$, reactor conversion suddenly drops to a low level despite the fact that the catalyst may still be relatively active.

The lower branch of the outer solution does not satisfy the initial condition (2.7); thus an inner solution is constructed near $a=1$ using the stretched variable

$$
\xi=(1-a) / \varepsilon
$$

and this is matched with the lower branch of the outer solution.

The upper and lower branches are connected through a branching inner solution, valid within a thin layer about the branching activity $a_{*}$ and of thickness determined largely by the residence time in the reactor. The stretched variable appropriate to the jump is

$$
\bar{a}=\left[a-a^{*}-\delta(\varepsilon)\right] / \varepsilon
$$

As will be shown in a subsequent section, this branching inner solution can be matched successfully only with the upper branch of the outer solution (where $A>A^{*}$ ); it fails to match with the lower branch. This failure is due to the existence of a distinguished (singular) limit which necessitates the analysis of another transition region across which the lower-branch outer solution can be matched with the branching inner solution [6]. It turns out that the proper stretched variable for this second transition expansion is

$$
a^{+}=\left[a-a^{*}-\rho(\varepsilon)\right] / \varepsilon^{2 / 3}
$$


This transition layer is much thicker than the first branching inner layer. The quantities $\delta(\varepsilon)$ and $\rho(\varepsilon)$ are shifts determined by matching.

The net result is that the concentration of reactant $A$ will be described as a continuous function of activity even across the jump. The situation is depicted in Figure 2, where it can be seen that the five solutions: I inner, II lower-branch outer,

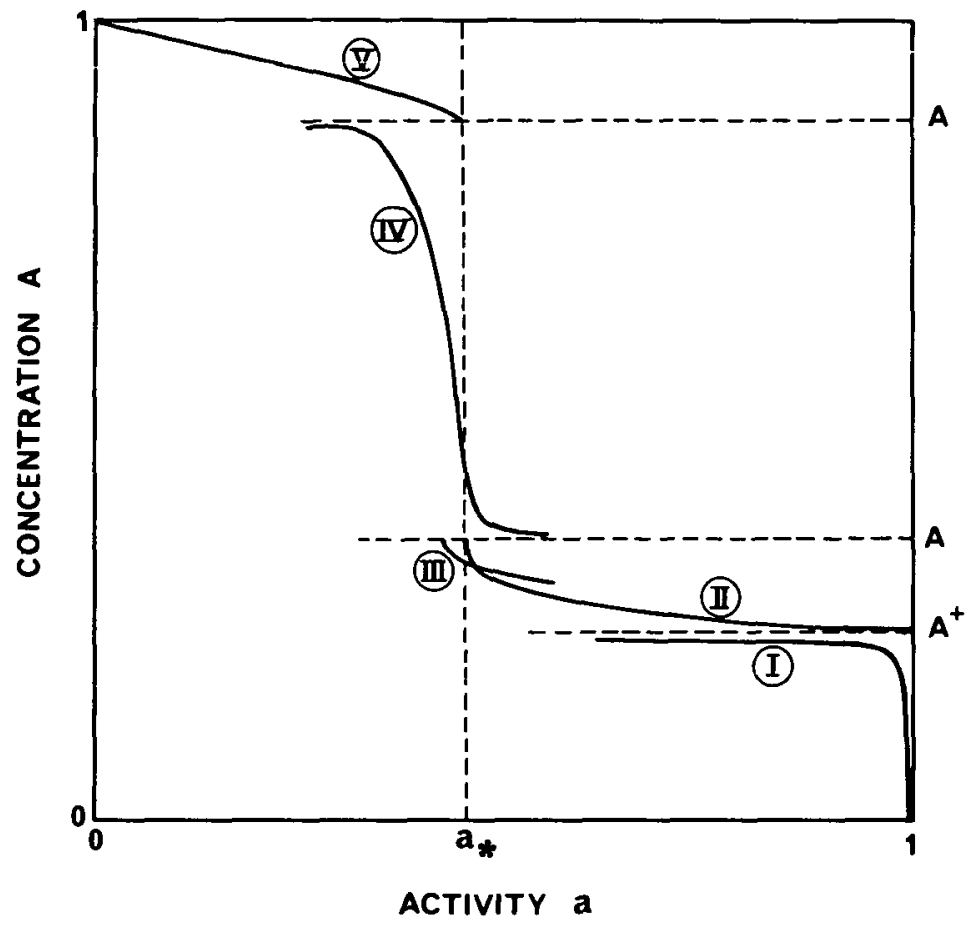

Fig. 2. Schematic of the five solutions in the phase plane.

III transition, IV branching inner and V upper-branch outer are all required to be determined and matched for a complete description of reactor performance.

\section{Outer solution}

The governing equation in the outer region is (2.5). We assume that $A$ has the following asymptotic expansion

$$
A(a, \varepsilon)=\tilde{A}_{0}(a)+\varepsilon \tilde{A}_{1}(a)+\ldots
$$

This leads to the following sequence of subproblems:

$$
1-\frac{H\left(1-\tilde{A}_{0}\right)\left(\beta+\gamma \tilde{A}_{0}+\tilde{A}_{0}^{2}\right)}{\tilde{A}_{0} a}=0
$$




$$
\frac{H\left[2 \tilde{A}_{0}^{3}+\tilde{A}_{0}^{2}(\gamma-1)+\beta\right] \tilde{A}_{1}}{a \tilde{A}_{0}^{2}}=\frac{d \tilde{A}_{0}}{d a} .
$$

The solutions are readily found to be

$$
\begin{gathered}
a=\frac{H\left(1-\tilde{A}_{0}\right)\left(\beta+\gamma \tilde{A}_{0}+\tilde{A}_{0}^{2}\right)}{\tilde{A}_{0}}=F\left(\tilde{A}_{0}\right) \quad \text { say, } \\
\tilde{A}_{1}(a)=-\frac{a \tilde{A}_{0}^{4}}{H^{2}\left[2 \tilde{A}_{0}^{3}+\tilde{A}_{0}^{2}(\gamma-1)+\beta\right]^{2}} .
\end{gathered}
$$

These solutions do not satisfy (2.7). Their asymptotic behaviour in the limit $a \rightarrow 1$ is required for later matching. Rewriting the variable $a$ in terms of $\xi$, we find that, as $\xi \rightarrow 0$,

$$
\begin{gathered}
A_{0} \sim A^{+}+\varepsilon \frac{A^{+2} \xi}{H\left[2 A^{+3}+A^{+2}(\gamma-1)+\beta\right]}+O\left(\varepsilon^{2}\right), \\
\tilde{A}_{1} \sim-\frac{A^{+4}}{H^{2}\left[2 A^{+3}+A^{+2}(\gamma-1)+\beta\right]^{2}}+O(\varepsilon),
\end{gathered}
$$

where $A^{+}$is the smallest root of $h(A, 1)=0$.

The outer solution (3.4) is multivalued in that it possesses two separate branches. On linearizing (2.5) about the solution (3.4) we find asymptotic stability if

$$
2 A^{3}+A^{2}(\gamma-1)+\beta>0 .
$$

There are two distinct (positive) branch points, $A_{*}$ and $A_{*}^{*}$, determined by solving (3.8) as an equality. The lower branch outer solution is valid in the domain $\left(a_{*}, 1\right)$ where

$$
a_{*}=H\left(1-A_{*}\right)\left(\beta+\gamma A_{*}+A_{*}^{2}\right) / A_{*}
$$

and concentration of reactant varies in the range $\left(A^{+}, A_{*}\right)$. The upper branch is valid in $\left(0, a_{*}\right)$ where concentration varies from $A^{*}$ to unity. In addition to being a root of (3.8), $A_{*}$ is also a double root of (3.9). Another root of (3.9) is $A^{*}$ and

$$
A^{*}=\beta / A_{*}^{2} \text {. }
$$

For later matching with the transition expansions to be constructed in the vicinity of $a_{*}$, we require the asymptotic behaviour of the lower and upper branches as $a \rightarrow a_{*}$. The lower branch of the outer solution as $a \rightarrow a_{*}$ is asymptotically

$$
\tilde{A}_{0} \sim A_{*}-\left[\frac{A_{*}\left(a-a_{*}\right)}{H\left(\frac{\beta}{A_{*}^{2}}-A_{*}\right)}\right]^{\frac{1}{t}}+\frac{\left(\frac{\beta}{A_{*}^{2}}\right)\left(a-a_{*}\right)}{2 H\left(\frac{\beta}{A_{*}^{2}}-A_{*}\right)^{2}}+\ldots
$$




$$
A_{1} \sim-\frac{\left(1-A_{*}\right)\left(\beta+\gamma A_{*}+A_{*}^{2}\right)}{4\left(\frac{\beta}{A_{*}^{2}}-A_{*}\right)\left(a-a_{*}\right)}-\frac{\left(1-A_{*}\right)\left(\beta+\gamma A_{*}+A_{*}^{2}\right)\left(\beta / A_{*}^{2}\right)}{4 \sqrt{ } H \sqrt{ } A_{*}\left(\frac{\beta}{A_{*}^{2}}-A_{*}\right)^{5 / 2}\left(a-a_{*}\right)^{\frac{1}{2}}}+\ldots
$$

while the upper branch has the following behaviour:

$$
\begin{gathered}
\tilde{A}_{0} \sim \frac{\beta}{A_{*}^{2}}+\frac{\left(\frac{\beta}{A_{*}^{2}}\right)\left(a_{*}-a\right)}{H\left(\frac{\beta}{A_{*}^{2}}-A_{*}\right)^{2}}-\frac{1}{H^{2}}\left(\frac{\beta}{A_{*}^{2}}\right) \frac{\left(\frac{\beta}{A_{*}^{2}}+A_{*}\right)\left(a_{*}-a\right)^{2}}{\left(\frac{\beta}{A_{*}^{2}}-A_{*}\right)^{5}}+\ldots \\
\tilde{A}_{1} \sim-\frac{\left(1-A_{*}\right)\left(\beta+\gamma A_{*}+A_{*}^{2}\right)\left(\beta / A_{*}^{2}\right)^{2}}{H A_{*}\left(\frac{\beta}{A_{*}^{2}}-A_{*}\right)^{4}}+\ldots
\end{gathered}
$$

\section{Inner solution}

We now obtain the solution valid near the boundary $a=1$. Using the stretched variable $\xi$ defined in (2.8) and assuming the following asymptotic expansion for reactant concentration,

$$
A \sim A_{0}(\xi)+\varepsilon A_{1}(\xi)+\ldots
$$

we obtain the equations

$$
\begin{gathered}
\frac{d A_{0}}{d \xi}=-h\left(A_{0}, 1\right), \\
A_{0}(0)=0,
\end{gathered}
$$

and

$$
\begin{gathered}
\frac{d A_{1}}{d \xi}=-A_{1} \frac{\partial h\left(A_{0}, 1\right)}{\partial A}+\xi \frac{\partial h\left(A_{0}, 1\right)}{\partial a} \\
A_{1}(0)=0
\end{gathered}
$$

at leading order and $O(\varepsilon)$, respectively, where

$$
\begin{gathered}
\frac{\partial h\left(A_{0}, 1\right)}{\partial A}=\frac{H\left[2 A_{0}^{3}+A_{0}^{2}(\gamma-1)+\beta\right]}{A_{0}^{2}} \\
\frac{\partial h\left(A_{0}, 1\right)}{\partial a}=\frac{H\left(1-A_{0}\right)\left(\beta+\gamma A_{0}+A_{0}^{2}\right)}{A_{0}} .
\end{gathered}
$$

The solutions to (4.2) and (4.4) are

$$
\int_{0}^{A_{0}(\xi)} \frac{x d x}{H(1-x)\left(\beta+\gamma x+x^{2}\right)-x}=\xi
$$


and

$$
A_{1}=\int_{0}^{\xi} s \frac{\partial h\left[A_{0}(S), 1\right]}{\partial a} \exp \left\{-\int_{s}^{\xi} \frac{\partial h\left[A_{0}(m), 1\right]}{\partial A} d m\right\} d S,
$$

with the following asymptotic behaviour in the limit $\xi \rightarrow \infty$,

$$
\begin{gathered}
A_{0}(\xi)=A^{+}+\mathrm{TST}, \\
A_{1}(\xi)=-\frac{A^{+4}}{H^{2}\left[2 A^{+3}+A^{+2}(\gamma-1)+\beta\right]^{2}}+\frac{A^{+2} \xi}{H\left[2 A^{+3}+A^{+2}(\gamma-1)+\beta\right]} .
\end{gathered}
$$

Here, TST denotes transcendentally small terms.

\section{Matching of inner and lower-outer solutions}

We define the following intermediate variable

$$
\theta=(1-a) / \eta
$$

where

$$
\varepsilon \ll \eta \ll 1 .
$$

The inner solution, written in terms of the intermediate variable, $\theta$, is

$$
\begin{aligned}
A \sim A^{+}-\frac{\varepsilon A^{+4}}{H^{2}\left[2 A^{+3}+A^{+2}(\gamma-1)+\beta\right]^{2}} & +\frac{A^{+2} \eta \theta}{H\left[2 A^{+3}+A^{+2}(\gamma-1)+\beta\right]} \\
& +O\left(\varepsilon^{2}\right)+O(\varepsilon \eta)+O\left(\eta^{2}\right)
\end{aligned}
$$

and this is identical with the lower branch of the outer solution written in the same variables. Clearly, the two expansions match and the common part is

$$
A^{+}-\frac{\varepsilon A^{+4}}{H^{2}\left[2 A^{+3}+A^{+2}(\gamma-1)+\beta\right]^{2}}+\frac{\varepsilon A^{+2} \xi}{H\left[2 A^{+3}+A^{+2}(\gamma-1)+\beta\right]}
$$

\section{Branching inner region}

In an attempt to join the lower and upper branches of the outer solution, we stretch the variable $a$ around the branch point according to

$$
\bar{a}=\left[a-a_{*}-\delta(\varepsilon)\right] / \varepsilon .
$$

This transforms (2.5) to

$$
\frac{d A}{d \bar{a}}=1-\frac{H(1-A)\left(\beta+\gamma A+A^{2}\right)}{A\left(a_{*}+\varepsilon \bar{a}+\delta(\varepsilon)\right)} .
$$


We now seek a solution of the form

$$
A(\bar{a} ; \varepsilon) \sim \bar{A}_{0}(\bar{a})+\varepsilon \bar{A}_{1}(\bar{a})+\ldots
$$

and this leads to the following $O(1)$ and $O(\varepsilon)$ equations:

$$
\begin{gathered}
\frac{d \bar{A}_{0}}{d \bar{a}}=h\left(\bar{A}_{0}, a_{*}\right) \\
\frac{d \bar{A}_{1}}{d \bar{a}}=\bar{A}_{1} \frac{\partial h\left(\bar{A}_{0}, a_{*}\right)}{\partial A}+\bar{a} \frac{\partial h\left(\bar{A}_{0}, a_{*}\right)}{\partial a}
\end{gathered}
$$

on the assumption that $\delta(\varepsilon) \ll \varepsilon$. We note that $a_{*}$ is defined in (3.9) and $A_{*}$ satisfies (3.8) under equality. Then (6.4) can be rearranged as

$$
\frac{d \bar{A}_{0}}{d \bar{a}}=\frac{H}{a_{*} \bar{A}_{0}}\left(\bar{A}_{0}-A_{*}\right)^{2}\left(\bar{A}_{0}-\beta / A_{*}^{2}\right)
$$

and we require the following asymptotic limits:

$$
\begin{array}{ll}
\bar{a} \rightarrow+\infty, & \bar{A}_{0} \rightarrow A_{*} ; \\
\bar{a} \rightarrow-\infty, & \bar{A}_{0} \rightarrow \beta / A_{*}^{2} .
\end{array}
$$

The solution to $(6.6)$ is

$$
\frac{\left(\beta / A_{*}^{2}\right)}{\left(\beta / A_{*}^{2}-A_{*}\right)^{2}} \ln \left(\frac{\bar{A}_{0}-\beta / A_{*}^{2}}{\bar{A}_{0}-A_{*}}\right)+\frac{A_{*}}{\left[\left(\beta / A^{2}\right)-A_{*}\right]\left(\bar{A}_{0}-A_{*}\right)}=\frac{H \bar{a}}{a_{*}},
$$

where the constant of integration has been absorbed into the quantity shift $\delta(\varepsilon)$.

The asymptotic behaviour of $\bar{A}_{0}(\bar{a})$ as $\bar{a} \rightarrow-\infty$ is

$$
\bar{A}_{0}(\bar{a}) \sim \beta / A_{*}^{2}+O\left(e^{\lambda \bar{a}}\right)
$$

where

$$
\lambda=\frac{H\left(\beta / A_{*}^{2}-A_{*}\right)^{2}}{\left(\beta / A_{*}^{2}\right) a^{*}}
$$

while for $\bar{a} \rightarrow+\infty$ we find that

$$
\bar{A}_{0}(\bar{a}) \sim A_{*}+\frac{\left(1-A_{*}\right)\left(\beta+\gamma A_{*}+A_{*}^{2}\right)}{\left(\beta / A_{*}^{2}-A_{*}\right) \bar{a}}+\frac{\beta\left(1-A_{*}\right)^{2}\left(\beta+\gamma A_{*}+A_{*}^{2}\right)^{2}}{A_{*}^{3}\left(\beta / A_{*}^{2}-A_{*}\right)^{3}} \frac{\ln \bar{a}}{\bar{a}^{2}}+\ldots
$$

We are not interested in the full solution for $\bar{A}_{1}$, but only in its asymptotic behaviour in the limits $\bar{a} \rightarrow \pm \infty$. By using the asymptotic behaviour of $\bar{A}_{0}$ as $\bar{a} \rightarrow \pm \infty$, we obtain the corresponding behaviour of $\bar{A}_{1}$ as follows: 


$$
\begin{gathered}
\bar{A}_{1}(\bar{a}) \sim \frac{A_{*} \bar{a}^{2}}{4 H\left(1-A_{*}\right)\left(\beta+\gamma A_{*}+A_{*}^{2}\right)}-\frac{\left(\beta / A_{*}^{2}\right) \bar{a} \ln \bar{a}}{6 H\left(\beta / A_{*}^{2}-A_{*}\right)^{2}}+\ldots \quad \text { as } \bar{a} \rightarrow+\infty ;(6.13) \\
\bar{A}_{1}(\bar{a}) \sim-\frac{\left(1-A_{*}\right)\left(\beta+\gamma A_{*}+A_{*}^{2}\right)\left(\beta / A_{*}^{2}\right)^{2}}{H A_{*}\left(\beta / A_{*}^{2}-A_{*}\right)^{4}}-\frac{\left(\beta / A_{*}^{2}\right) \bar{a}}{H\left(\beta / A_{*}^{2}-A_{*}\right)^{2}}+\ldots \quad \text { as } \bar{a} \rightarrow-\infty .
\end{gathered}
$$

By using the intermediate variable

$$
\theta=\left[a-a^{*}-\delta(\varepsilon)\right] / \eta
$$

in which $\eta$ meets the condition (5.2), it is easy to see that the branching inner solution automatically matches with the upper branch of the outer solution. However, matching with the lower branch fails because the branching inner solution exhibits only algebraic decay in the limit $\bar{a} \rightarrow+\infty$. The implication of the failure is the existence of another layer and a separate expansion in which the matching is carried out.

\section{Transition expansion}

We define the following stretched variable

$$
a^{+}=\left[a-a_{*}-\rho(\varepsilon)\right] / v(\varepsilon)
$$

in which the shift quantity $\rho(\varepsilon)$ and the scaling factor $v(\varepsilon)$ are to be determined. The concentration $A$ is assumed to possess the following local expansion

$$
A \sim A_{*}+\mu_{1}(\varepsilon) A_{1}\left(a^{+}\right)+\mu_{2}(\varepsilon) A_{2}\left(a^{+}\right)+\ldots .
$$

Combining (7.1) and (7.2) with (2.5) and noting that

$$
h\left(A_{*}, a_{*}\right)=\frac{\partial h\left(A_{*}, a_{*}\right)}{\partial A}=\frac{\partial^{2} h\left(A_{*}, a_{*}\right)}{\partial A \partial a}=0
$$

leads to the equation

$$
\begin{aligned}
& \frac{\varepsilon \mu_{1}}{v} \frac{d A_{1}\left(a^{+}\right)}{d a^{+}}+\frac{\varepsilon \mu_{2}(\varepsilon)}{v} \frac{d A_{2}\left(a^{+}\right)}{d a^{+}}+\ldots \\
& \quad=v a^{+} \frac{\partial h}{\partial a}\left(A_{*}, a_{*}\right)+\frac{1}{2} \mu_{1}^{2} A_{1}^{2} \frac{\partial^{2} h}{\partial A^{2}}\left(A_{*}, a_{*}\right)+\rho(\varepsilon) \frac{\partial h\left(A_{*}, a_{*}\right)}{\partial a} \\
& \quad+\mu_{1} \mu_{2} A_{1} A_{2} \frac{\partial^{2} h}{\partial A^{2}}\left(A_{*}, a_{*}\right)+\frac{\mu_{1}^{3}}{3 !} A_{1}^{3} \frac{\partial^{3} h\left(A_{*}, a_{*}\right)}{\partial A^{3}}+\ldots
\end{aligned}
$$

The first three principal terms balance if

$$
\varepsilon \mu_{1} / v=v=\mu_{1}^{2}
$$


with the result that

$$
\begin{aligned}
\mu_{1} & =\varepsilon^{1 / 3}, \\
\nu & =\varepsilon^{2 / 3} .
\end{aligned}
$$

The remaining terms balance for

$$
\mu_{2}=\varepsilon^{2 / 3},
$$

and we are led to the following subproblems:

$$
\begin{gathered}
\frac{d A_{1}}{d a^{+}}=a^{+} \frac{\partial h_{*}}{\partial a}+\frac{1}{2} A_{1}^{2} \frac{\partial^{2} h_{*}}{\partial A^{2}}, \\
\frac{d A_{2}}{d a^{+}}=A_{1} A_{2} \frac{\partial^{2} h_{*}}{\partial A^{2}}+\frac{1}{6} A_{1}^{3} \frac{\partial^{3} h_{*}}{\partial A^{3}},
\end{gathered}
$$

where

$$
\begin{aligned}
\frac{\partial^{2} h_{*}}{\partial A^{2}} & =-\frac{2\left(\beta / A_{*}^{2}-A_{*}\right)}{\left(1-A_{*}\right)\left(\beta+\gamma A_{*}+A_{*}^{2}\right)}<0, \\
\frac{\partial h_{*}}{\partial a} & =\frac{A_{*}}{H\left(1-A_{*}\right)\left(\beta+\gamma A_{*}+A_{*}^{2}\right)}>0 \\
\frac{\partial^{3} h}{\partial A^{3}} & =\frac{6 \beta}{A_{*}^{3}\left(1-A_{*}\right)\left(\beta+\gamma A_{*}+A_{*}^{2}\right)}>0 .
\end{aligned}
$$

Equation (7.9) can be rewritten as

$$
\frac{d A_{1}}{d a^{++}}=-A_{1}^{2}+K a^{++}
$$

where

$$
a^{++}=\left[-\frac{1}{2} \frac{\partial^{2} h^{*}}{\partial A^{2}}\right] a^{+}
$$

and

$$
K=\left(\partial h^{*} / \partial a\right) /\left(-\frac{1}{2} \frac{\partial^{2} h^{*}}{\partial A^{2}}\right)^{2}
$$

This equation is of Ricatti type and can be solved by the transformation

$$
A_{1}=\frac{1}{V_{1}} \frac{d V_{1}}{d a^{++}}
$$

then (7.14) becomes

$$
\frac{d^{2} V_{1}}{d a^{++2}}-K a^{++} V_{1}=0
$$


Define

$$
\overline{\bar{a}}=K^{1 / 3} a^{++}=\left[\frac{A_{*}\left(\beta / A_{*}^{2}-A_{*}\right)}{H\left(1-A_{*}\right)^{2}\left(\beta+\gamma A_{*}+A_{*}^{2}\right)^{2}}\right]^{1 / 3} a^{+} .
$$

Then (7.18) reduces to

$$
\frac{d^{2} V_{1}}{d \bar{a}^{2}}-\bar{a} V_{1}=0,
$$

which is the familiar Airy equation. Solutions can be written in terms of modified Bessel functions [1].

$$
V_{1}= \begin{cases}c \sqrt{ }(\overline{\bar{a}}) K_{1 / 3}\left(\frac{2}{3} \overline{\bar{a}}^{3 / 2}\right), \quad \overline{\bar{a}}>0 \\ \frac{\pi c}{\sqrt{ } 3} \sqrt{ }-\overline{\bar{a}}\left\{J_{1 / 3}\left[2 / 3(-\overline{\bar{a}})^{3 / 2}\right]+J_{-1 / 3}\left[2 / 3(-\overline{\bar{a}})^{3 / 2}\right]\right\}, \quad \overline{\bar{a}}<0 .\end{cases}
$$

To match with the lower branch of the outer expansion we require the asymptotic behaviour of this transition solution in the limit $\overline{\bar{a}} \rightarrow \infty$. This is

$$
\begin{aligned}
A_{1}(\overline{\bar{a}}) \sim c\left\{\left[\frac{A_{*}\left(\beta / A_{*}^{2}-A_{*}\right)}{H\left(1-A_{*}\right)^{2}\left(\beta+\gamma A_{*}+A_{*}^{2}\right)^{2}}\right]^{1 / 6} \sqrt{a^{+}}\right. \\
\left.\quad+\frac{1}{4}\left[\frac{H\left(1-A_{*}\right)^{2}\left(\beta+\gamma A_{*}+A_{*}^{2}\right)^{2}}{A_{*}\left(\beta / A_{*}^{2}-A_{*}\right)}\right]^{1 / 3} \frac{1}{a^{+}}+\ldots\right\} .
\end{aligned}
$$

\section{Matching of transition and lower branch outer solutions}

Define an intermediate variable

$$
\theta=\left[a-a_{*}-\rho(\varepsilon)\right] / \eta,
$$

where $\varepsilon^{2 / 3} \ll \eta \ll 1$. Combining (3.11) and (3.12) and rewriting in terms of the intermediate variable $\theta$ gives

$$
\begin{aligned}
A(\theta) \sim A_{*} & -\left[\frac{A_{*} \eta \theta}{H \Lambda}\right]^{\frac{1}{2}}+\frac{\left(\beta / A^{2}\right) \eta \theta}{2 H \Lambda^{2}}-\frac{\left(1-A_{*}\right) \Delta \cdot \varepsilon}{4 \Lambda \eta \theta} \\
& -\frac{\left(1-A_{*}\right) \Delta \cdot\left(\beta / A_{*}^{2}\right)}{4 H^{\frac{t}{3}} A_{*}^{\frac{1}{*}} \Lambda^{5 / 2}} \cdot \frac{\varepsilon}{(\eta \theta)^{\frac{1}{2}}}-\left[\frac{A_{*}}{4 H \Lambda \eta \theta}\right]^{\frac{1}{2}} \rho(\varepsilon) \\
& +\frac{\rho(\varepsilon) \cdot\left(\beta / A_{*}^{2}\right)}{2 H \Lambda^{2}}+\varepsilon \frac{\left(1-A_{*}\right) \Delta \rho(\varepsilon)}{4 \Lambda \eta^{2} \theta^{2}} \\
& +\frac{\left(1-A_{*}\right) \Delta\left(\beta / A_{*}^{2}\right) \varepsilon \rho(\varepsilon)}{8\left(H A \Lambda^{5} \eta^{3} \theta^{3}\right)^{\frac{1}{2}}}+\ldots
\end{aligned}
$$


where

$$
\Delta=\beta+\gamma A_{*}+A_{*}^{2}
$$

and

$$
\Lambda=\beta / A_{*}^{2}-A_{*}
$$

The transition expansion, written in terms of $\theta$, is

$$
A \sim A_{*}+c\left[\frac{A_{*} \Lambda}{H\left(1-A_{*}\right)^{2} \Delta^{2}}\right]^{1 / 6} \sqrt{ } \eta \theta+\frac{c}{4} \cdot\left[\frac{H\left(1-A_{*}\right)^{2} \Delta^{2}}{A_{*} \Lambda}\right]^{1 / 3} \frac{\varepsilon}{\eta \theta}+\ldots
$$

Equations (8.2) and (8.5) match at leading order when

$$
c=-\left[\frac{A_{*}\left(1-A_{*}\right) \Delta}{H \Lambda^{2}}\right]^{1 / 3}
$$

and $A_{1}\left(a^{+}\right)$has the following form

$$
A_{1}\left(a^{+}\right) \sim-\left[\frac{A_{*} a^{+}}{H \Lambda}\right]^{\frac{1}{2}} \frac{\left(1-A_{*}\right) \Delta}{4 \Lambda a^{+}}+\ldots
$$

for $a^{+} \rightarrow \infty$. This can be used in (7.10) and the result integrated to obtain an expression for $A_{2}\left(a^{+}\right)$, valid in the limit $a^{+} \rightarrow \infty$

$$
A_{2}\left(a^{+}\right) \sim \frac{\left(\beta / A_{*}^{2}\right)}{2 H \Lambda^{2}} a^{+}+\frac{\left(\beta / A_{*}^{2}\right)\left(1-A_{*}\right) \Delta}{2 H^{\frac{1}{2}} \Lambda^{5 / 2} A_{*}^{\frac{1}{*}}} \frac{1}{\sqrt{ } a^{+}}+\ldots
$$

When (8.7) and (8.8) are combined and written in terms of $\theta$, the result is

$$
A \sim A_{*}-\left[\frac{A_{*} \eta \theta}{H \Lambda}\right]^{\frac{1}{2}}+\frac{\left(\beta / A_{*}^{2}\right) \cdot \eta \theta}{2 H \Lambda^{2}}-\frac{\varepsilon\left(1-A_{*}\right) \Delta}{4 \Lambda^{*} \eta \theta}+\frac{\varepsilon}{\sqrt{\eta \theta}} \cdot \frac{\left(\beta / A_{*}^{2}\right)\left(1-A_{*}\right) \Delta}{2 H^{\frac{1}{2}} A_{*}^{\frac{1}{2}} \Lambda^{5 / 2}}+\ldots
$$

In order for this to match with (8.2) we must have

$$
\rho(\varepsilon)=-\frac{3}{2} \cdot \frac{\left(\beta / A_{*}^{2}\right)\left(1-A_{*}\right) \Delta}{A_{*} \Lambda^{2}} \varepsilon
$$

The asymptotic form of $A_{1}\left(a^{+}\right)$in the limit $a^{+} \rightarrow a_{0}^{+}<0$, where $a_{0}^{+}$is the first zero of the Airy function, is readily seen to be

$$
A_{1}\left(a^{+}\right) \sim \frac{\left(1-A_{*}\right) \Delta}{\Lambda\left(a^{+}-a_{0}^{+}\right)}+\ldots
$$

and substituting this result into (7.10) and solving for $A_{2}\left(a^{+}\right)$gives

$$
A_{2}\left(a^{+}\right) \sim \frac{\left(\beta / A_{*}^{2}\right)\left(1-A_{*}\right)^{2} \Delta^{2}}{A_{*} \Lambda^{3}} \cdot \frac{\ln \left(a^{+}-a_{0}^{+}\right)}{\left(a^{+}-a_{0}^{+}\right)^{2}}+\ldots,
$$


where

$$
a_{0}^{+}=-2.338107\left[\frac{H\left(1-A_{*}\right)^{2} \cdot \Delta^{2}}{A_{*} \Lambda}\right]^{1 / 3} .
$$

These expressions will be needed in the matching of the transition, and branching inner expansions to follow.

\section{Matching of transition and branching inner solutions}

We write the branching inner variable as

$$
\bar{a}=\frac{1}{\varepsilon^{1 / 3}}\left[a^{+}-a_{0}^{+}-\sigma(\varepsilon)\right],
$$

where $a_{0}^{+}$is defined in (8.13) and

$$
\sigma(\varepsilon)=-a_{0}^{+}-\frac{\rho(\varepsilon)}{\varepsilon^{2 / 3}}+\frac{\delta(\varepsilon)}{\varepsilon^{2 / 3}},
$$

where $\rho(\varepsilon)$ has been obtained in (8.10). Define the intermediate variable as

$$
\theta=\frac{a^{+}-a_{0}^{+}-\sigma(\varepsilon)}{\eta}
$$

Then the transition expansion (7.2), with $A_{1}\left(a^{+}\right)$and $A_{2}\left(a^{+}\right)$given in (8.11) and (8.12), respectively, when written in terms of this intermediate variable is

$$
\begin{aligned}
A^{+}(\theta) \sim A_{*} & +\frac{\varepsilon^{1 / 3} \cdot\left(1-A_{*}\right) \Delta}{\Lambda \eta \theta}-\frac{\varepsilon^{1 / 3} \sigma(\varepsilon)\left(1-A_{*}\right) \Delta}{\Lambda \eta^{2} \theta^{2}} \\
& +\varepsilon^{2 / 3} \frac{\left(\beta / A_{*}^{2}\right)\left(1-A_{*}\right)^{2} \cdot \Delta^{2}}{A_{*} \Lambda^{3}} \cdot \frac{\ln (\theta \eta)}{\eta^{2} \theta^{2}}+\ldots
\end{aligned}
$$

Similarly, the branching inner expansion for $\vec{a} \rightarrow \infty$ assumes the form

$$
\begin{aligned}
\bar{A}(\theta) \sim A_{*} & +\frac{\varepsilon^{1 / 3}\left(1-A_{*}\right) \Delta}{\Lambda \cdot \eta \theta}-\frac{\left(\varepsilon^{2 / 3} \ln \varepsilon\right)\left(\beta / A_{*}^{2}\right)\left(1-A_{*}\right)^{2} \Delta^{2}}{3 A_{*} \Lambda^{3} \eta^{2} \theta^{2}} \\
& +\varepsilon^{2 / 3} \frac{\left(\beta / A_{*}^{2}\right)\left(1-A_{*}\right)^{2} \Delta^{2}}{A_{*} \Lambda^{3}} \cdot \frac{\ln (\eta \theta)}{\eta^{2} \theta^{2}}+\ldots
\end{aligned}
$$

when written in terms of $\theta$.

Equations (9.4) and (9.5) agree if

$$
\sigma(\varepsilon)=\frac{\left(\beta / A_{*}^{2}\right)\left(1-A_{*}\right) \Delta \varepsilon^{1 / 3} \ln \varepsilon}{3 A_{*} \Lambda^{2}}
$$


and $\delta(\varepsilon)$ can be calculated from (9.2). The result is

$$
\begin{aligned}
\delta(\varepsilon)= & -2.338 \varepsilon^{2 / 3}\left[\frac{H\left(1-A_{*}\right)^{2} \Delta^{2}}{A_{*} \Lambda}\right]^{1 / 3} \\
& -\frac{3}{2} \cdot \frac{\left(\beta / A_{*}^{2}\right)\left(1-A_{*}\right) \Delta}{A_{*} \Lambda^{2}} . \varepsilon-\frac{\left(\beta / A_{*}^{2}\right)\left(1-A_{*}\right) \Delta}{3 A_{*} \Lambda^{2}} . \varepsilon \ln (1 / \varepsilon)<0 .
\end{aligned}
$$

Thus, we find that $\rho(\varepsilon)$ and $\delta(\varepsilon)$ are both strictly negative so that the jump occurs at a value of $a$ a little less than $a_{*}$. In terms of time, if the branching time is $t_{0}$, then $A$ will jump to another state a little after $t_{0}$. The quantity shift $\rho(\varepsilon)$ turns out to be $O(\varepsilon)$. This means that in order to obtain the full correction $A_{2}\left(a^{+}\right)$in (7.2) we must return to (7.10) and include the forcing term $\partial h / \partial a$ which has been omitted in going from (7.4) to (7.10). The additional term which results, must automatically match with the lower branch of the outer solution written in terms of the appropriate intermediate variable.

In the case of the shift $\delta(\varepsilon)$, we have apparently omitted not only a forcing term $O(\varepsilon)$ but also two other larger terms $O\left(\varepsilon^{2 / 3}\right)$ and $O(\varepsilon \ln 1 / \varepsilon)$. These corrections must be included by inserting terms of this order between $\bar{A}_{0}$ and $\bar{A}_{1}$ in (6.3); the result will be two further equations to be solved for the corrections. The corrections must match automatically with similar terms in the upper branch outer solution written in the appropriate intermediate variable. The additional terms resulting from both $\rho(\varepsilon)$ and $\delta(\varepsilon)$ must also match across the transition and branching inner regions. All of this affects the solutions beyond leading order but the quantity shifts necessarily remain unaltered, and are correct as given here.

\section{References}

[1] M. Abramowitz and I. A. Stegun, Handbook of mathematical functions (Dover Publications, New York, 1970).

[2] R. Aris, "Phenomena of multiplicity, stability and symmetry", Annals New York Acad. Sci. 231 (1974), 86-98.

[3] R. Aris, The mathematical theory of diffusion and reaction in permeable catalysts, Vols. I and II (Clarendon Press, Oxford, 1975).

[4] D. D. Bruns, J. E. Bailey and D. Luss, "Steady state multiplicity and stability of enzymatic reaction systems", Biotechnol. and Bioeng. 15 (1973), 1131-1145.

[5] J. D. Buckmaster, A. K. Kapila and G. S. S. Ludford, "Linear condensate deflagration for large activation energy", Acta Astro. 3 (1976), 593-614.

[6] J. D. Cole, Perturbation methods in applied mathematics (Blaisdell Publishing Co., Mass., 1968).

[7] A. L. Devera and A. Varma, "Substrate-inhibited enzyme reaction in a tubular reactor with axial dispersion", Chem. Eng. Sci. 34 (1979) 275-278.

[8] D. D. Do and R. H. Weiland, "Consistency between rate expressions for enzyme reactions and deactivation", Biotechnol. and Bioeng. (1980) (in press).

[9] R. Haberman, "Slowly varying jump and transition phenomena associated with algebraic bifurcation problems", SIAM J. Appl. Math. 37 (1979) 69-106. 
[10] T. C. Ho, "Uniqueness criteria of the steady state in automotive catalysis", Chem. Eng. Sci.31 (1976), $235-240$.

[11] A. K. Kapila, "Reactive-diffusive system with Arrhenius kinetics: dynamics of ignition", SIAM J. Appl. Math. (1980) (in press).

[12] A. K. Kapila, "Arrhenius systems: dynamics of jump due to slow passage through criticality", (1980) unpublished manuscript.

[13] A. K. Kapila and B. J. Matkowsky, "Reactive-diffusive system with Arrhenius kinetics: multiple solutions, ignition and extinction", SIAM J. Appl. Math. 36 (1979), 373-389.

[14] D. R. Kassoy, "Extremely rapid transient phenomena in combustion, ignition and explosion", SIAM-AMS Proceedings 10 (1976), 61-72.

[15] A. Liñán and F. A. Williams, "Theory of ignition of a reactive solid by constant energy flux", Combustion Sci. Tech. 3 (1971), 91-98.

[16] A. Liñán and F. A. Williams, "Ignition of a reactive solid exposed to a step in surface temperature", SI AM J. Appl. Math. 36 (1979), 587-603.

[17] C. J. Periera and A. Varma, "Uniqueness criteria for the steady state in automotive catalysis", Chem. Eng. Sci. 33 (1978), 1645-1657.

\section{Department of Chemical Engineering $\dagger$}

University of Queensland

St. Lucia 4067

† D. D. Do and R. H. Weiland are now with the Departments of Chemical Engineering at California Institute of Technology, Pasadena, CA 91125, and Clarkson College of Technology, Potsdam, NY 13676 , respectively. 